\title{
EL CONSEJO DE LA UNIÓN EUROPEA COMO FORO INTERGUBERNAMENTAL DE LA TOMA DE DECISIONES
}

\author{
JOSU DE MIGUEL BÁRCENA
}

Universidad del País Vasco / Euskal Herriko Unibertsitatea 


$$
\text { " }
$$




\section{EL CONSEJO DE LA UNIÓN EUROPEA COMO FORO INTERGUBERNAMENTAL DE LA TOMA DE DECISIONES}

POR

JOSU DE MIGUEL BÁRCENA*

Universidad del País Vasco / Euskal Herriko Unibertsitatea

\section{INTRODUCCIÓNY FUNDAMENTOS BÁSICOS PARA EL ANÁLISIS}

El complejo institucional desarrollado dentro de la Unión Europea (en adelante UE), responde sin lugar a dudas a la exclusividad y originalidad del proceso de integración comunitaria, que ha ido configurando un sistema de organización política que supera las concepciones tradicionales adscritas a una organización internacional clásica y el propio Estado contemporáneo'.

EI Consejo de la Unión Europea (en adelante CUE), es la institución central sobre la que gravita el proceso decisorio en la UE. Las facultades legislativas del CUE se expresan de una manera muy amplia al aludir el art. 202 del Tratado de la Comunidad Europea (en adelante TCE), a la disposición "de un poder de decisión». Los Tratados ori-

* Josu de Miguel Bárcena es Profesor de Derecho Constitucional en la Universidad del País Vasco / EHV.

1 A este respecto, ver RuIPÉREz, J.: La Constitución europea y la teoría del Poder Constituyente: Algunas reflexiones críticas desde el derecho político. Biblioteca Nueva, Madrid, 2000, y MARTín Y Pérez DE NANCLARES, J.: El sistema de competencias de la Comunidad Europea, McGraw-Hill, Madrid, 1997, págs. 1-21. 
ginarios también atribuyeron al CUE funciones ejecutivas que sin embargo deben ser delegadas en la Comisión. No obstante, la principal función del CUE es alcanzar y, posteriormente, formalizar acuerdos entre los Estados miembros, que más tarde son elevados a la categoría de decisiones².

La originalidad del CUE estriba, en que en virtud del principio de representatividad territorial que encarna ${ }^{3}$, se compone de miembros de ejecutivos que ejercen una labor legislativa en el plano comunitario y ejecutiva de sus propias decisiones en el plano interno. Su composición netamente estatal y la importancia de las acciones gubernamentales y legislativas que realiza en pos de la consecución de los objetivos comunitarios, le presuponen como un contrapeso intergubernamental a la esencia y labor supranacional de otras instituciones comunitarias ${ }^{4}$, léase Comisión o Tribunal de Justicia de las Comunidades Europeas.

Asimismo, los miembros del CUE actúan tanto en el ámbito de competencias de los Tratados comunitarios, como en el ámbito de

2 Según Pescatore, el CUE «...es el verdadero legislador de la Comunidad; un legislador, hay que subrayarlo, de carácter no parlamentario, y cuya base de legitimidad es, desde luego, internacional y no directamente popular". PESCATORE, P.: "El ejecutivo comunitario. Justificación del cuatripartidismo instituido por los Tratados de Roma y París", en Derecho de Integración, n. ${ }^{\circ} 25,1977$.

3 La naturaleza original del propio Consejo, desde la fundación de la Comunidad hasta la cuarta ampliación, respondia a una combinación de diversos parámetros jurídicos y políticos. A saber, el principio de igualdad soberana (presencia de todos los Estados miembros) y el principio de efectividad (ponderación moderada del voto en función de la población). MANGAS MARTín, A.: "Democracia y eficacia en la Unión Europea ampliada: el restablecimiento de los equilibrios globales en el sistema de votaciones del Consejo», en Gaceta Jurídica, B-112, abril de 1996, pág. 6.

4 Supranacionalidad entendida para nosotros como dos planos diferentes; un plano inferior que hace referencia a un sistema de producción normativa que ope$\mathrm{ra}$ independientemente de los Estados miembros, el cual sustenta y es sustentado por un proceso decisorio dinámico, no jerárquico y abierto a diferentes tipos de cooperación en orden a dar solución a los problemas políticos que se presenten y otro plano superior, donde la supranacionalidad es entendida como una premisa para sustituir el orden liberal internacional, por un régimen destinado a imponer una nueva disciplina al estrecho interés nacional-estatal y a rebajar los excesos relacionados con el mismo en la sociedad internacional. A este respecto ver WEILER, J.: "Demos, telos and the German Maastricht decision", Harvard Jean Monnet Working Paper, http://www.jeanmonnetprogram.org/papers/, 1995; WEILER, J. H. H.: The Constitution of Europe. Do the new clothes have an Emperor? And others essays on European Integration, en Cambridge University Press, Cambridge, 1999. Sobre la supranacionalidad en la propia UE, ver SOMEK, A.: "On Supranationality", en European Integration Online Papers, n. ${ }^{\circ}$ 3, VI. 5, 2001; http://eiop.or.at/eiop/ 
actuación concertada o de la cooperación entre los países miembros en materias presumiblemente ajenas a la competencia de la Comunidad, es decir, en el área intergubernamental. El carácter de tratadomarco del TCE posibilita la ampliación de las primeras, mientras que el resto de las actuaciones se desarrollan alli donde no puede llegar la actuación comunitaria ${ }^{5}$.

Cabe contemplar al CUE además, dadas sus capacidades legislativas, como un foro supraestatal donde se satisface la legitimidad en el plano comunitario y estatal, entendida parcialmente ésta como la capacidad efectiva de un sistema de gobierno de satisfacer el bienestar y las demandas materiales realizadas por la ciudadanía. Así, el CUE se puede haber convertido en una institución donde los Estados mancomunan esfuerzos y competencias, en orden a manejar variables económicas y políticas que progresivamente han ido escapando al control estatal dada la interdependencia de los regímenes globales ${ }^{6}$.

El objetivo de este artículo es cuestionarnos la dimensión real del proceso de integración comunitaria, llevando a cabo un análisis centrado en el proceso decisorio desarrollado dentro del CUE. Los puntos de vista más convencionales, entienden que el CUE, por su estructura estatal y funcionamiento, es una institución o foro interestatal donde los Estados acotan en lo posible la progresiva integración comunitaria7, bien a través de la representación de sus pro-

5 GONZÁLEZ SANCHEZ, E.: "La evolución institucional de la Unión Europea: del sistema cuatripartito previsto en los tratados originarios, a un sistema institucional tripartito en la perspectiva de realización de la unificación europea», en Revista de Instituciones Europeas, VI, 21, n. ${ }^{\circ} 1,1994$.

6 Ver WESSELS, W.: "The EC Council:The Community's Decisionmaking Center", en KEOHANE, R. O., y HofFMAN, S. (ed.): The new European Community: Decisionmaking and institutional change. Westview, Colorado, 1991, págs. 134-5. También PAYCE, R., y WESSELS, W.: "The search for an ever closer Union: a framework for analysis", en PrYCE, R. (ed.): The dynamics of European Union, Trans-European Policy Studies Association, Londres, 1987, págs. 4-6.

7 De esta opinión, por ejemplo, Host., M.: «Negotiations in the European Union: the hidden influence on Council", en Columbia International Affairs Online, wwwc.cc.columbia.edu/sec/dic/ciao/isa/isa.html, octubre de 2000; KEOHANE, R. O., y HOFFMAN, S.: "Institutional Change in Europe in the 1980's", en KEOHANE, R. O., Y Hoffman, S.: (ed.), op. cit., y Moravsick, A.: «Preferences and Power in the European Community: A Liberal Intergovernmentalist Approach", en Journal of Common Market Studies (JCMS) VI. 31, n. ${ }^{\circ}$, diciembre de 1993. Los análisis más jurídicos realizados en torno a la naturaleza y funcionamiento del CUE, revelan generalmente esta dimensión del propio CUE, aunque no eluden la posibilidad de que otros procesos y elementos distorsionen esta percepción. Ver por ejemplo Sobrino, J. M.: "El marco comunitario de la participación de las Comunidades Autónomas en los 
pios intereses o bien otorgando legitimidad a la integración positiva como delimitadora de la integración negativa que con regularidad propone e intenta llevar a cabo la propia Comisión ${ }^{8}$. Esta afirmación resulta en parte cierta, sobre todo en las áreas de cooperación intergubernamental, como es el caso de la Política Exterior y de Seguridad Común (en adelante PESC) y en los ámbitos de la Cooperación en Asuntos de Justicia e Interior (en adelante JAl) definitivamente no integrados.

Sin embargo, nuestra intención es presentar una imagen alternativa del CUE y por tanto del propio proceso de integración comunitaria. Desde la perspectiva jurídica, tomarán importancia elementos como la distancia entre la capacidad material y formal del CUE como institución legislativa, debido tanto a la creciente absorción de responsabilidades y especialización de los cuerpos inferiores e intermedios que lo conforman, es decir Comité de Representantes Permanentes (en adelante COREPER) y los grupos de trabajo, como al relativo desplazamiento de la responsabilidad legislativa hacia el Parlamento Europeo (en adelante PE) y el propio Consejo Europeo.

Por otro lado, haremos referencia al proceso político que posibilita la llegada a acuerdos entre los diferentes participantes en el proceso decisorio dentro del CUE. Un proceso político, globalmente influido por la paulatina instauración de un contexto negociador (el de la UE) enfocado a la solución de problemas comunes, por la creciente asimilación de normas y prácticas representativas del método comunitario en la cultura organizativa del propio CUE y por la elevada tecnificación y despolitización de muchas de las materias legisladas en el ámbito comunitario.

La validez de nuestro acercamiento está condicionado por la necesidad de impulsar un análisis que disuelva la reiterativa distinción entre alta y baja política que caracterizan los análisis relativos al pro-

Consejos de Ministros de la Unión European, en Instituto VAsCo de Administración PúBliCA: La participación de las Comunidades Autónomas en los Consejos de Ministros de la Unión Europea, Bilbao, 1998.

8 Sobre los conceptos de integración positiva y negativa, ver SCHARPF, F.W.: "Negative and positive integration in the political economy of European Welfare States", en MARKS, G.; ScharPF, F. W.; SchmitTer, P. C., y StREeck, W. (eds.): Governance in the European Union, Sage Publications, Londres, 1996, y OfFE, C.: "Democracia y Estado de Bienestar: un régimen europeo bajo la tensión de la integración europea", en Zona Abierta, 92/93, año 2000, págs. 254-256. 
ceso de integración europea ${ }^{9}$ y a la importancia de reconsiderar todas nuestras afirmaciones bajo el prisma e influencia de las modificaciones institucionales realizadas en el Tratado de Niza, con la entrada de nuevos Estados oteando en el horizonte.

\section{CONSEJO DE LA UNIÓN EUROPEAY AUTORIDAD LEGISLATIVA: ELEMENTOS PARA UNA CONSIDERACIÓN ALTERNATIVA}

EI CUE es una institución única, aunque dividida funcionalmente por áreas o políticas. Para llevar a cabo sus tareas, el CUE se organiza internamente a partir de la Presidencia, el COREPER y los diferentes comités y los grupos de trabajo, con el fundamental apoyo logístico de la Secretaría General. El desarrollo histórico del CUE, se caracteriza por un progresivo aumento de los Consejos sectoriales tanto en número de reuniones como en tipo de formaciones ${ }^{10}$. La especialización y el crecimiento cuantitativo de las reuniones y los asuntos tratados dentro del CUE, son un perfecto indicador del progresivo e imparable traspaso de competencias por parte de los Estados miembros a la UE y de las prioridades políticas por las que se decanta el proyecto comunitario".

Como señalamos con anterioridad, el art. 202 delTCE atribuye al CUE, en su formación de Ministros o representante equivalente de

9 En el ámbito comunitario, la alta política ha hecho tradicionalmente referencia a las materias relacionadas con la PESC o JAl, núcleo o sustancia de la soberanía estatal, de ahí su tratamiento y consideración intergubernamental dentro de las políticas de UE; sin embargo, encontramos anacrónico denominar baja política a las materias definitivamente integradas bajo el régimen comunitario, como es el caso de la cesión de instrumentos económicos y monetarios en particular, en un período de gran interdependencia y globalización económica como el que vivimos. Nos parece oportuno recordar además, que la creación y regulación de la moneda es por ejemplo una competencia originalmente concedida al poder soberano. Así lo vio el propio Bodino, en Bodino, J.: Los VI Libros de la República, Centro de Estudios Constitucionales, Madrid, 1992. En especial el Libro Primero, Capítulo X, pág. 372.

10 Sobre estas dos cuestiones ver Morata, F: La Unión Europea: Procesos, actores y políticas, Ariel Ciencia Política, Barcelona, 1998, pág. 152. La lista oficial de las formaciones del CUE figura actualmente en el DOCE, serie C 174, de 23 de junio de 2000, pág. 1.

1 HaYes-REnshaw, F: "The European Council and the Council of Ministers", en Cram, L.; Dinan, D., y Nugent, N. (eds.): Developments in the European Union, St. Martin's Press, New York, 1999, pág. 27. 
rango ministerial, el poder de decisión para garantizar la consecución de los fines establecidos en el propio Tratado. Sin embargo, el poder legislativo del CUE se ve mediatizado por diferentes actores y procesos, de carácter interinstitucional e intrainstitucional.

\subsection{El incremento externo de responsabilidades y centros decisores: Parlamento y Consejo Europeo}

En primer lugar, tanto el PE como el Consejo Europeo, han ido asumiendo $\mathrm{e}$ incrementando responsabilidades legislativas y funcionales en detrimento del propio CUE. Éste es menos independiente en las áreas referidas al primer pilar comunitario, en primer lugar porque casi siempre depende de la iniciativa normativa de la Comisión para llevar a cabo su labor legislativa y en segundo lugar, porque las enmiendas constitucionales realizadas en los diferentes Tratados, han logrado involucrar cada vez más al PE en el proceso decisorio comunitario ${ }^{12}$.

Así, el Tratado de la Unión Europea (en adelante TUE), aunque limitado materialmente, convierte por fin al PE en un autentico legislador dentro de la UE a través del procedimiento de codecisión ${ }^{13}$.Y aunque no a los ojos de los más ortodoxos, el Tratado de Amsterdam enmendó de forma seria el mismo procedimiento ${ }^{14}$, suprimiendo la posibilidad de que el CUE actúe de forma unilateral tras la falta de acuerdo en la conciliación de segunda lectura, acrecentando la paridad de ambas instituciones y posibilitando de esta manera la expansión del núcleo decisor comunitario en su vertiente institucional ${ }^{15}$. El

12 Para un breve acercamiento empírico sobre esta cuestión, ver HIx, S.: The Political System of the European Union, St. Martin's Press, New York, 1999, págs. 94-96.

13 De interés, ver MuYLLE, K.: "ls the European Parliament a Legislator?", en European Public Law, VI, 6, Issue 2, 2000; NAVArro Bastida, N.: Parlamento Europeo y poder normativo en la Unión Europea, Universidad de Salamanca, Salamanca, 1995, y PIoueras Garcia, A. J.: Participación del Parlamento Europeo en la actividad legislativa comunitaria, Granada, Universidad de Granada, 1993.

14 Ver Mangas Martín, A.: "La reforma institucional en el Tratado de Amsterdam", en Revista de Derecho Comunitario Europeo (RDCE), n. ${ }^{\circ}$, enero/junio de 1998, pág. 16, y ROLDÁN BARBERO, J.: "El Sistema Institucional: el nuevo y el pendiente", en Noticias de la UE, n.`186, julio de 2000, pág. 20.

15 El procedimiento de codecisión II, enmendado en el Tratado de Amsterdam, aunque no afecta al tamaño del núcleo legislativo que generan ambas ins- 
Tratado de Niza, aunque modestamente, ha vuelto a implicar al PE dentro del proceso decisorio comunitario, ampliando entre otras cuestiones, la capacidad material en la que el PE legisla a través del procedimiento de codecisión ${ }^{16}$.

Por otra parte, el Consejo Europeo, reunión de los Jefes de Estado y de Gobierno de los Estados miembros de la UE17, lleva a cabo una labor entre lo funcional y legislativo, que ha restado además carácter intergubernamental a la labor realizada por el propio CUE. Además de proporcionar impulsos generales, a modo de declaraciones o reclamaciones de carácter político para el desarrollo general del proceso comunitario, el Consejo Europeo tiene atribuidas funciones decisorias expresamente reconocidas por los Tratados en el ámbito delTítulo VII delTCE, es decir, en la política económica y monetaria de la UE (arts. 99, 112, 113, 121 y 122 delTCE). El Consejo Europeo tiene también un papel decisivo como origen e impulsor de los package deal o paquetes de negociación que más tarde son desarrollados y negociados dentro del CUE.

Asimismo, el Consejo Europeo realiza una labor clave en la planificación y desarrollo del la PESC, bien a través de orientaciones generales y estrategias comunes sobre la misma (art. 13 del TUE) o bien como último recurso al interés nacional vital, en lo que se ha venido a denominar la "versión modificada del Compromiso de Luxemburgo" (art. 23.2 del TUE) ${ }^{18}$. Desde una perspectiva más general, estos son

tituciones, ya que se sigue requiriendo el acuerdo de las dos instituciones para generar actos comunitarios, sin embargo acrecienta la discreción de la Comisión como "administrador de las políticas comunitarias y delTJCE como interprete estatutario». El procedimiento de codecisión II eliminó la posibilidad de una cuarta lectura por parte del CUE después del primer veto del PE. EI CUE podía sacar adelante su posición común por mayoría cualificada y sólo un segundo veto por mayoría absoluta del PE, paralizaría de forma definitiva el proceso decisorio. Una vez eliminada esta posibilidad, se evita una situación tirante entre el PE y el CUE. Así lo ven Garret, G., y TSEbelis, G.: "The institutional foundations of intergovernmentalism and supranationalism in the EU", International Organization, VI, 55, n. ${ }^{\circ} 2,2001$.

16 TCE, arts. 13.2 (no discriminación), 67.5 (Título IV delTratado), 137.2 (disposiciones sociales), 157.3 (acciones para fomentar la cohesión económica y social) y 191 (partidos políticos europeos).

17 Para un acercamiento más amplio sobre el origen, naturaleza y funciones del Consejo Europeo, recomendamos ver BULMER, S.: «The European Council and the Council of European Union: Shapers of a European Confederation", en Publius, VI, 26, n. 4, 1996; Johnston, M. T.: The European Council: Gatekeeper of the European Community. Westview Press, Colorado, 1994, y WeRTs, J.: The European Council. North Holland, Amsterdam, 1992. 
mecanismos de cierre intergubernamental que permiten a los Estados ostentar el control final sobre decisiones en ámbitos de cooperación política dentro de la propia UE. Sin embargo, en lo referente al desarrollo institucional, parece haberse instaurado una implicita división de tareas entre el CUE y el propio Consejo Europeo. EI CUE, en cualquiera de las formaciones determinadas, tiende a esquivar las cuestiones más conflictivas a la espera de que sea el próximo Consejo Europeo el que resuelva los problemas en última instancia, ya que debido a su composición, parece hallarse en condiciones de superar la presunta fragmentación del CUE y la saturación de tareas del Consejo de Asuntos Generales.

Dentro del CUE, el proceso decisorio está condicionado por la fragmentación material correspondiente a las diferentes políticas sobre las que legisla la propia UE, por un paulatino desvío de responsabilidades legislativas hacia el COREPER y grupos de trabajo y por un proceso político donde prima generalmente el consenso y la viabilidad del propio sistema.

\subsection{Fragmentación material y rivalidad administrativa en los órganos de preparación de los trabajos legislativos del CUE: el COREPER y los Comités del CUE}

EI COREPER es un órgano formado por los Jefes de las Representaciones Permanentes de los Estados y sus adjuntos. Se encarga de examinar y negociar las proposiciones remitidas por la Comisión y actúa en calidad de filtro político entre los grupos de trabajo y el CUE ${ }^{19}$. En el mismo nivel del COREPER, existen los denominados Comités del CUE, con una función bastante similar al propio COREPER,

18 En este sentido, ver DEVUYST, Y.: «The Community-Method after Amsterdam", en JCMS, VI, 37, n.॰1, 1999, pág. 114; algunos autores consideran que el Tratado de Amsterdam institucionalizó e introdujo dentro del Derecho comunitario el Compromiso de Luxemburgo a través del párrafo segundo del art. 11 delTCE, referente a la cooperación reforzada. Esta situación, parece haberse reconducido a través de la nueva redacción propuesta por el Tratado de Niza para dicho artículo. Ver MARTÍNez SierRa, J. M.: "La cooperación reforzada tras Niza", en Revista de las Cortes Generales, $2 .^{\circ}$ semestre de 2001 , págs. 61 y 62.

19 EI COREPER se divide en dos secciones o niveles. EI COREPER I se compone de los Representantes Permanentes adjuntos y prepara los Consejos tradicionalmente tildados como técnicos (educación, cultura, pesca, energía, investigación, etc.). Sus trabajos los ha preparado el denominado Grupo Mertens. EI COREPER II está compuesto por los Embajadores Permanentes de los Estados 
es decir, asesorar al CUE y a la Comisión y preparar las reuniones del primero ${ }^{20}$. La fragmentación administrativa y la división material del trabajo entre los órganos inferiores al pleno de los Consejos sectoriales, facilita la asignación y el cumplimiento de las tareas encomendadas a los mismos. Sin embargo, la distinción de políticas a través de los pilares comunitarios, origina rivalidades administrativas y solapa competencias entre el COPERER y los distintos comités creados para el tratamiento de las diferentes políticas ${ }^{21}$. El diseño institucional y el proceso decisorio adscrito a las políticas de cooperación intergubernamental, pueden servir de ejemplo para ilustrar esta cuestión.

EITUE previó la posibilidad de crear un comité de coordinación compuesto por altos funcionarios, en lo que respecta a las políticas derivadas de la Cooperación en Asuntos de Justicia e Interior (art. 23). Así, el citado comité tendría que "...contribuir, sin perjuicio de lo dispuesto en el art. 207 delTCE, a la preparación de los trabajos del Consejo en las materias que se refiere el art. 29». La formalización de este párrafo ha ayudado a coordinar y delimitar los trabajos entre el COREPER y el Comité de Coordinación de Cooperación Jurídica y Policial. Mientras que este último contribuye a la preparación de los trabajos que maneja el COREPER, es decir, realiza una tarea eminentemente técnica, el COREPER lleva a cabo una tarea más política ${ }^{22}$. Sin embargo,

miembros, discuten temas de mayor calado político y preparan los Consejos de Asuntos Generales, economia y finanzas y los asuntos relacionados con la PESC y la JAI. Sus trabajos los prepara el Grupo Antici. Sobre el COREPER en particular, ver Sobrino, J. M., op. cit.; Mangas Martin, A.: El Comité de Representantes Permanentes de las Comunidades Europeas, Centro de Estudios Constitucionales, Madrid, 1980, y DiNAN, D.: Ever Closer Union? An introduction to the European Community, McMillan, Londres, 1994, págs. 250 y 251.

20 Entre los Comités más importantes, el Comité Especial de Agricultura, creado en julio de 1962, el Comité del art. 133, encargado de las política comercial de la UE, el Comité Presupuestario, el Comité Económico y Financiero, el Comité de Empleo, formado por un número paritario de representantes públicos y privados o el Comité político, dedicado a las cuestiones relacionadas con la PESC. Sobre la relación de Comités situados al mismo nivel del COREPER, ver HAYES-RENSHAW, F., op. cit., págs. 28-30.

21 En este sentido, ver un artículo donde se describe los métodos de trabajo tanto de COREPER como de los diferentes Comités y las distintas rivalidades corporativas y administrativas entre los mismos; LEWIS, J.: "The methods of community in EU decision-making and administrative rivalry in the Council's infraestructure", en Journal of European Public Policy (JEPP), 7: 2, junio de 2000.

22 VilariÑo PINTOS, E.: "La cooperación en ámbitos de justicia e interior en el Tratado de la UE. Los aspectos básicos para su realización", en Revista de Instituciones Europeas, VI, 21, n. ${ }^{\circ}$, año 1994, págs. 79-80. 
se ha constatado que el COREPER II, el cuerpo que filtra los informes de dicho Comité al CUE, frecuentemente se encuentra con duplicación de tareas y competencias entre ambos cuerpos ${ }^{23}$.

En lo referente a la PESC, el COREPER y el Comité Político no están exentos de la competencia intraministerial -en realidad ambos están compuestos por representantes de los Ministerios de Asuntos Exteriores-, sobre todo tras la reciente creación de un Comité Político provisional, asesor permanente para el Alto Representante de la PESC y Secretario General del CUE y la nueva Política Exterior Común de Defensa y Seguridad, Javier Solana. El nuevo Comité Politico y de Seguridad ${ }^{24}$, con competencias ampliadas al ámbito de la seguridad y la defensa y constituido por altos funcionarios con sede en Bruselas, a efectos se supone de posibilitar su convocatoria mucho más frecuente y en un plazo más corto, tiene previsto reemplazar al propio Comité Político, con orígenes en la Cooperación Política Europea. Si bien es cierto que algunos Ministerios de Asuntos Exteriores no ven con malos ojos el traslado de algunas tareas a Bruselas en materia de PESC, también es verdad que los Embajadores Permanentes, conocedores y forjadores del proceso político dentro del CUE, no contemplan esta posibilidad como apropiada, ya que en cierto modo pierden el control sobre un proceso decisorio basado en la confianza y el conocimiento real de los procedimientos y las partes ${ }^{25}$.

A las descoordinaciones corporativas entre diferentes Consejos sectoriales ${ }^{26}$, se le pueden unir entonces cuestiones como las anteriormente descritas, que pueden afectar a la realización de políticas que requieran el uso de competencias vinculadas a más de un pilar comunitario, como es el caso de la PESC. La efectividad de ciertas decisiones o medidas dependerá de variables como la coherencia y el factor

23 WESTLAKE, M.: The Council of European Union, en Cartermill, Londres, 1995, pág. 239

24 La propuesta de crear un Comité de este tipo fue realizada por Italia y Gran Bretaña. Ver Financial Times, n. ${ }^{\circ} 15$, septiembre de 1999. Ahora es realidad tras Decisión del Consejo, DOCE L 49, 22 de febrero de 2000.

25 Significativa, en este sentido, una entrevista realizada a un miembro de la Comisión. Ver LEWIS, J.: "The methods of community in EU decision-making and administrative rivalry in the Council's infraestructure", op. cit., pág. 280.

26 No es de extrañar, en este sentido, presenciar contradicciones o conflictos en materia de gasto agrícola entre el Consejo de Agricultura y el ECOFIN y entre este último y el de Medio Ambiente, en materia de infraestructuras. Sobre estas cuestiones, ver PETERSON, J., y BOMBERG, E.: Decision-Making in the European Union, St. Martin Press, Nueva York, 1999. 
tiempo, que pueden verse resentidas por una relación competitiva entre los Comités y el propio COREPER y por una estructura organizativa excesivamente fragmentada $y$ compleja de cara a una negociación efectiva ${ }^{27}$.

\subsection{Apuntes para la controversia: el CUE como legislador virtual de la UE}

EI COREPER es el centro sobre el que gravita el proceso decisorio dentro del CUE. Es el lugar donde las dimensiones horizontales $y$ verticales de coordinación del CUE se entrecruzan ${ }^{28}$. En el nivel superior, el COREPER es el encargado de preparar las reuniones de los Consejos de Ministros sectoriales; inferiormente, se encarga de coordinar los distintos grupos de trabajo que comienzan a preparar y examinar las propuestas que más tarde serán de nuevo revisadas y finalmente aprobadas por el CUE. Los grupos de trabajo son reuniones de expertos pertenecientes a las Representaciones Permanentes de los Estados miembros ${ }^{29}$ o bien provenientes de las capitales; algunos tienen carácter permanente y otros se crean ad hoc en función de las necesidades del momento. La doctrina maneja un número de entre 200 y 250 grupos de trabajo, teniendo una frecuencia de reuniones variable: algunos se reúnen periódicamente y otros de forma esporádica y siempre en función de la agenda del propio COREPER.

En los ámbitos definitivamente integrados, la Comisión detenta la iniciativa legislativa que desencadena el diálogo CUE-Comisión como tándem iniciador y elaborador de la producción normativa de la UE. La Comisión no se desentiende de su propuesta ni la propuesta es para el CUE un mero trámite que le permita decidir. La razón es

27 En cualquier caso, esta conclusión no debe estar sujeta a generalizaciones, ya que en algunos casos, el tiempo y la experiencia han conseguido delimitar con cierta claridad las competencias y las tareas entre algunos Comités y el propio COREPER. Es el caso de este último y el propio Comité Especial de Agricultura.

28 EDWARDS, G.: «National Sovereignity vs integration?The Council of Ministers", en RiCHARDson, J. (ed.): European Union: Power and Policy-Making, Routledge, Londres, 1996, pág. 136.

29 Sobre la Representaciones Permanentes de los Estados miembros, ver Hayes-Renshaw, F.; Leouesne, C., y Mayor López, P.: «The Permanent Representations of the Member States to the European Communities", en JCMS, VI, 28, n. ${ }^{\circ}$, diciembre de 1989. 
que aquella está facultada a modificar su propuesta inicial mientras el CUE no haya decidido y éste último no puede enmendar las propuestas de la Comisión más que por la unanimidad de todos sus miembros. La Comisión puede realizar una gran labor como gestor de la negociación y conciliación de los diferentes intereses estatales en pos del logro de una mayoría que permita llevar adelante la propuesta legislativa, entre otras razones porque existen lazos orgánicos entre el CUE y la Comisión, al permitirsele a ésta última una presencia permanente en las sesiones del Consejo, COREPER y grupos de trabajo ${ }^{30}$.

El manejo y la elaboración de propuestas dentro del CUE sigue una lógica subsidiaria de responsabilidades. Las propuestas de la Comisión son parcialmente manejadas dentro de los grupos de trabajo, COREPER y los Ministros, en función del nivel técnico y la sensibilidad política que caracterice el tema tratado. Los grupos de trabajo llevan a cabo una labor que en buena medida es similar a la descrita como fase pre - negociadora en la literatura especializada ${ }^{31}$. Realizan un examen de la propuesta en concreto y elaboran un informe en el que destacan los aspectos de la propuesta inicial sobre los que se ha llegado a un acuerdo, los denominados puntos I del informe. Los puntos II son los aspectos sobre los que no hay acuerdo, $y$ por lo tanto se someten a discusión dentro del COREPER. Éste, al examinar el informe, ratifica, si lo cree procedente, los puntos I que ya fueron adoptados por las delegaciones a nivel de grupos de trabajo. Sobre los aspectos en los que persiste el desacuerdo, el COREPER emplea su capacidad de debate y comienza asi la fase técnico política. El diálogo en el COREPER I y II adquiere márgenes de flexibilidad y amplias posibilidades de transacción, debido a los contactos direc-

30 La presencia de la Comisión está asegurada a través de los arts. 5.2 y 5.3 del Reglamento interno del CUE (en adelante R.i.). EI nuevo R. i. del CUE fue establecido en Decisión de CUE de 5 de junio de 2000, 2000/396/CE, CECA, EURATOM. Puede verse en el Diario Oficial de las Comunidades Europeas (en adelante DOCE), serie $L$ 149, de 23 de junio de 2000, págs. 20-35.

31 Hampson y Stein distinguen dos estadios o fases más o menos diferenciadas en todo proceso negociador. La fase pre-negociadora, descrita esencialmente como una fase de diagnóstico y definición de los problemas y acuerdos básicos, y las denominadas como negociaciones formales, donde los participantes intercambian información, discuten directamente e intercambian posturas en pos de un acuerdo. HAMPSON FEN, O., y HART, M.: Multilateral negotiations: Lessons from Arms Control, Trade and Environment, The J. Hopkins University Press, Baltimore, 1995, y STEIN, J. G. (ed.): The Processes of international Prenegotiations, The J. Hopkins University Press, Baltimore, 1997. 
tos que los Representantes Permanentes $y$ sus Adjuntos mantienen con sus Gobiernos ${ }^{32}$.

Las deliberaciones en el seno del CUE, hablamos a nivel ministerial, se desarrollan conforme a un orden del día preparado por el COREPER y dividido en dos partes, la parte A y la parte B lart. 3.7 del R. i. del CUE). Los puntos incluidos en la parte A del orden del día del Consejo, son aquellos temas en los que el COREPER recomienda a los Ministros que los aprueben de forma global y automática, sin entrar en discusión; cuando el COREPER incluye una propuesta en la parte $B$, recomienda al Consejo que delibere y decida sobre una propuesta que no reúne la mayoria necesaria de las delegaciones ni en los grupos de trabajo ni en el COREPER. En las áreas comunitarizadas, el porcentaje de temas o partes $A$ y $B$ del orden del dia, puede variar conforme a la singularidad del proceso político que acompaña la adopción de decisiones en las diferentes políticas ${ }^{33}$.

Además, es muy común que el itinerario que tenga que recorrer una propuesta hasta llegar a ser punto $A$ pueda ser muy diverso. Este tipo de puntos o temas $A$ son denominados por de Zwaan como falsos puntos $A$ y pueden constatarse en diferentes procedimientos 0 prácticas $^{34}$. Puede que en varias ocasiones anteriores fueran objeto de discusión como temas o puntos $B$, por lo que el acuerdo fue alcanzado en grupos de trabajo y COREPER un tiempo después y propuesto para formalizar como decisión en el propio CUE como punto A. Otro tipo de temas o puntos falsos $A$ son aquellos en los que de antemano se sabe que se ha llegado a un acuerdo extensivo pero incompleto en el seno del COREPER y grupos de trabajo; el Consejo en su formación de Ministros, se convierte entonces en órgano auxiliar o preparador de temas específicos, ya que el COREPER y los grupos de trabajo reclaman de forma implícita su orientación para llegar a un acuerdo formal $y$ definitivo.

32 Una clara descripción sobre todas estas cuestiones, en MANGAS MARTín, A., y LiÑAN Nogueras, D. J.: Instituciones y Derecho de la Unión Europea, McGrawHill, Madrid, 1996, págs. 280 y ss.

33 Por ejemplo, el Comité Especial de Agricultura, encargado de preparar los trabajos para el Consejo de Agricultura, es consciente de lo proclives que son los Ministros a negociar cara a cara o personalmente las propuestas legislativas que deben ser aprobadas en el seno del CUE, lo que se traduce en un aumento significativo de temas B con respecto a otros Consejos sectoriales. CuLLEY, P.: uThe Agricultural Council», en WESTLAKE, M.: The Council of European Union, op. cit.

34 DE ZWAAN, J.: The Permanent Representatives Committee: Its Role in European Union Decision-Making, Elsevier, Amsterdam, 1995, pág. 136. 
También se reconoce la existencia de temas o puntos falsos $\mathrm{B}^{35}$. Son propuestas en los que se ha llegado a un acuerdo total tanto en el COREPER como en los grupos de trabajo; sin embargo, cualquier Ministro de un Estado miembro puede sentir la necesidad de que se explicite su desacuerdo con la propuesta aprobada a nivel del Consejo de Ministros, lo que en cierta forma le puede servir para salvar la cara ante la opinión pública de su propio Estado, si existiera un malestar manifiesto ante una decisión adoptada que pueda ser considerada contraria a los intereses de ese Estado.

Los arts. 202 y 203 delTCE, evidencian que el poder de decisión corresponde al Consejo en su formación de Ministros o nivel equivalente (rango ministerial). Sin embargo, algunas cifran muestran una débil capacidad formal de decisión del CUE en su nivel más elevado. ¿Qué volumen de legislación es discutida realmente por los Ministros en las reuniones del CUE?

El estudio de Van Schendelen, enmarcado en el Consejo de Ministros de Agricultura, muestra que solamente un $13 \%$ de la legislación pasa por las manos de los Ministros en su preparación y posterior aprobación ${ }^{36}$. Aunque la doctrina maneja un porcentaje variable ${ }^{37}$, atendiendo a las diferentes formaciones sectoriales esa franja puede incluso disminuir si el Consejo en cuestión decide delegar parte de la legislación al Consejo Europeo, para que éste formule algunas

35 RÖHL, H. C.: «Die Beteiligung der Bundesrepublik Deutschland an der Rechtsetzung im Ministerrat der EU", en Europa Recht, 1994, pág. 426; también DE ZWAAN, J., op. cit., pág. 138.

36 VAN SCHENDELEN, M.C.P.M.: "The Council Decides": Does the Council Decides?", en JCMS, VI, 34, n. ${ }^{\circ} 4$, diciembre de 1996 . Así, un $65 \%$ de la legislación era pasada como temas $A$, es decir, los acuerdos se formalizaban en grupos de trabajo y COREPER y salvo excepciones contadas (0'93\%), se aprobaban sin discusión por los Ministros. Del $35 \%$ de material legislativo restante, es decir, los temas $B$, menos de la mitad era aprobado por los Ministros en alguna de sus reuniones y el resto se reenviaba a los cuerpos inferiores para ser negociados o tratados desde un nuevo punto de vista, es probable incluso que volviese a nivel ministerial formando parte de nuevos paquetes de temas o puntos $A$.

37 Hayes-Renshaw y Wallace, señalan que un $70 \%$ de la legislación es aprobada en los niveles de grupos de trabajo y que entre un 15 y $20 \%$ es aprobada en el COREPER, por lo que los Ministros manejan y aprueban entre una franja del 10 y $15 \%$ de legislación, ver HAYES-Renshaw, F, y WALLACE, H.: The Council of Ministers, en Macmillan Press, Londres 1997, pág. 40. Wessels señala de forma más genérica que un $80 \%$ de las decisiones las toman formalmente el «aparato burocrático" del CUE. Ver WESSELS, W.: "The EC Council: The Community's Decisionmaking Center», pág. 140. 
directrices generales que faciliten el trabajo de los grupos de trabajo y COREPER.

Estas cifras muestran una distancia entre la capacidad legislativa de jure y de facto del CUE. Juridicamente, ninguno de los órganos auxiliares del propio CUE está habilitado para formalizar definitivamente legislación comunitaria ${ }^{38}$, sin embargo, las anteriores cifras dan a entender el extraordinario peso del COREPER y los grupos de trabajo en el aparato decisor comunitario. Por un lado, esta circunstancia da pie a realizar apuntes sobre una progresiva marginalización del CUE en el conjunto decisorio comunitario ${ }^{39}$, algo que en nuestra opinión requiere un análisis estructural más detallado referido a los diferentes Consejos sectoriales y al estilo decisorio que caracteriza a la división por pilares de las políticas comunitarias, es decir, las políticas definitivamente integradas por un lado y las políticas estrictamente de cooperación por otro.

Por otro lado, sin obviar otras cuestiones problemáticas vinculadas al fenómeno anteriormente descrito ${ }^{40}$, creemos que la sustantiva delegación de responsabilidades legislativas por parte de los Ministros tanto en el COREPER como en los grupos de trabajo, en cierta

38 Sentencia de 23.2.1988, TJCE, Comisión c. Consejo, C-25/94. El Consejo no siempre venía cumpliendo esta formalidad. En ocasiones, confirmaba decisiones que en teoría eran tomadas por el COREPER. Asi, el TJCE anuló una Decisión del CUE, que atribuía a los Estados miembros el derecho de voto en el seno de la FAO, en relación con un acuerdo sobre recursos pesqueros en alta mar, debido a que el CUE nunca adoptó la Decisión, sino que confirmó una decisión anterior del COREPER. Los fundamentos de derecho 26 y 27 de la Sentencia, «...el Coreper no es una Institución de las Comunidades dotada por el Tratado de competencias propias, sino que constituye un órgano auxiliar del Consejo que efectúa, para este último, tareas de preparación y ejecución". Asimismo, "la función de ejecución de las tareas encomendadas por el Consejo, no faculta al Coreper para ejercitar el poder de decisión que corresponde, conforme al Tratado, al Consejon. En este sentido, señalar que el nuevo R. i. del CUE, reconoce al Coreper la posibilidad de tomar decisiones de procedimiento, en algunos casos tan importantes como la de publicar el resultado de votaciones o hacer un debate público del propio CUE.

39 Van Schendelen citando a KAPTEYN, P. J. G., y otros: Inleiding tot het recht van de Europese Gemeenschappen, Kluwer, Deventer, 1995. En VAN SCHENDELEN, M.C.P.M., op. cit., pág. 543.

40 Nos referimos en este caso, al peso real de la burocracia en el sistema decisorio de la UE y a la debilidad de los controles jurisdiccionales que sobre ella puedan operar. Sobre esta cuestión, ver RUBıo LLORENTE, F:: «El futuro político de Europa. El déficit democrático de la Unión Europea», en Claves de Razón Práctica, n. ${ }^{\circ}$ 90, marzo de 1999, pág. 29. 
manera intensifica la distancia entre el cuerpo legislativo concreto (en este caso los ciudadanos de los Estados, representados por sus Ministros) y la toma de decisiones dentro de la UE. No hay que olvidar que los Ministros, dentro del CUE, no forman parte de un órgano ejecutivo, donde la delegación de responsabilidades puede ser comprensible y hasta sana, sino que forman parte de un aparato legislativo y que por tanto representan a la ciudadanía de su Estado como cuerpo legislativo dentro de la UE. Sin embargo, cuestiones como la falta de trasparencia o la ausencia de control democrático sobre difusos centros decisores, en este caso eminentemente burocráticos, pueden ser solventados mediante propuestas que ayuden a mejorar el perfil democrático de la propia UE ${ }^{41}$.

\subsection{Dimensiones constitutivas del proceso político que envuelve la toma de decisiones dentro del CUE: consenso, despolitización $y$ factor institucional}

El poder de decisión dentro del CUE se expresa y se articula a través de los procedimientos de votación que prevén los Tratados. Se distinguen como procedimientos la mayoría simple, la mayoría cualificada y la unanimidad, si bien los propios Tratados contienen disposiciones en la que tanto la mayoría cualificada como la unanimidad puedan verse amplificadas por diversos requerimientos (art. 22 deITCE y art. 6.1 delTUE). Sin embargo, la disciplina y el comportamiento de los actores con respecto al voto dentro del CUE, no está siempre determinada por las provisiones legales de los Tratados. Diversos factores, bien de carácter político o bien institucional y sometidos a un grado de formalidad extremadamente flexible, confluyen en la configuración de un proceso político donde en la mayoría de las ocasiones prima la

41 De esta forma, los representantes de los Estados miembros dentro del CUE, podrían ser elegidos en sus Estados de origen de forma separada a los Gobiernos nacionales, pudiendo asi tener una dedicación plena y un control absoluto sobre el proceso legislativo dentro del CUE y expandiendo sobremanera la posibilidad de control por parte de la ciudadanía. El CUE se convertiría entonces en una forma concreta de senado, algo que en cierta forma ya es. Sobre esta cuestión, ZÜRN, M.: "Democratic Governance Beyond the Nation - State: The EU and Other International Institutions", en European Journal of International Relations, VI, 6 (2). 2000, págs. 204-205. Desde una perspectiva más genérica, ver ATINNA, F: «Integrazione e democracia: Un'analisi evoluzionista dell'Unione Europea», en Rivista Italiana di Scienza Politica, año XXX, n. 2 , agosto 2000. 
subsistencia del propio sistema, en detrimento de las expectativas estratégicas de los propios Estados. Veamos alguno de ellos.

El análisis del voto y la toma de decisiones como agregación de intereses dentro del CUE, es importante pero no determinante para llegar a entender $y$ es su caso conceptualizar el proceso decisorio dentro del mismo. El procedimiento de votación a través de la mayoría cualificada dentro del CUE, es estimable en un $10 \%$ sobre el total de las decisiones adoptadas ${ }^{42}$. El Compromiso de Luxemburgo ${ }^{43}$, un acuerdo informal de más que dudoso carácter legal, institucionalizó una forma de tomar decisiones basada fundamentalmente en el consenso entre los participantes. Si bien es verdad que con anterioridad a la formalización del Compromiso, el voto por mayoría era algo bastante inusual y que la invocación explícita del mismo, apenas superó la docena de ocasiones durante más de veinte años, lo cierto es que dentro del CUE se instauró una cultura del veto que obligaba a tomar las decisiones por unanimidad, aunque los Tratados hubiesen previsto lo contrario en las materias que se estuviese elaborando una propuesta.

Tradicionalmente, se ha considerado que el Compromiso de Luxemburgo ralentizó y convirtió en ineficaz el proceso decisorio comunitario en el período previo al Acta Única Europea 44 , sin embargo, análisis más recientes demuestran que el sistema decisorio comunitario se mostró eficaz en cuanto al tiempo de elaboración y la cantidad de legislación aprobada por el CUE durante ese período ${ }^{45}$. Hay que

42 Muchos de los Ministros y representantes de los estados miembros que participan en las reuniones de los Consejos sectoriales, nunca han presenciado la existencia de voto real o a "mano alzada" en los mismos, WESSELS, W.: «The EC Council: The Community's Decisionmaking Center", en KEOHANE, R. O., y HOFFMAN, S. (ed.), op. cit., pág. 147; a pesar de esta circunstancia, el peso del voto está en la mente de todos los participantes que toman parte en los diferentes niveles del proceso decisorio dentro del CUE, ver MOBERG, A.: "The voting system in the Council of the European Union. The balance between large and small countries», en Scandinavian Political Studies, VI, 21, n. ${ }^{\circ} 4$, 1998, pág. 349

43 Sobre el surgimiento y la incidencia concreta del Compromiso de Luxemburgo en el sistema comunitario, ver TEASDALE, A. L.: "The Life and Death of the Luxembourg Compromise", en JCMS, VI.31, n. ${ }^{\circ}$, diciembre de 1992.

44 Un acercamiento desde esta perspectiva, KRISLOV, S.; EHLERMANN, K. D., y WeILER, J. H. H.: «The Political Organs and the Decision-Making Process in the United States and the European Communityn, en CAPELLETTI, M.; SECOMBE, M., y WeILer, J. H. H. (eds.), Integration through Law. Europe and the American Federal Experience, Walter de Gruyter, Florencia 1986.

45 Asi lo demuestran SLOOT, T., y VerRSCHUREN, P.: “Decision-making Speed in the European Community", en JCMS, VI, 39, ${ }^{\circ}{ }^{\circ} 1,1990$. Golub también minimi- 
tener en cuenta además, que gracias a la mutación competencial operada en su favor durante la década de los setenta, la UE expandió su ámbito material de legislación a cuestiones como el medio ambiente, la educación o la energía.

EI AUE, en un movimiento constitucional cuyas consecuencias no fueron capaces de proyectar al futuro algunos Estados, estableció de forma generalizada el voto por mayoría dentro del CUE en el ámbito del mercado único (art. 95 delTCE). Podemos afirmar, sin temor a equivocarnos, que dicha medida transformó el proceso decisorio de la UE, al neutralizar la cultura del veto instaurada por el Compromiso de Luxemburgo. EI CUE sigue negociando y adoptando las decisiones de forma consensuada, sin embargo, no es lo mismo negociar la aprobación de una norma bajo la sombra de la mayoria cualificada, que bajo la sombra de la unanimidad ${ }^{46}$. El instrumento del veto permitía a algunos Estados miembros persistir en posiciones contrarias a la mayoria y la mayoría cualificada flexibiliza el proceso decisorio, en la medida que el recurso a la votación puede dejar fuera de juego a los Estados miembros contrarios a tomar una decisión en concreto. De esta manera, la mayoría cualificada resulta un eficaz instrumento psicológico, que conduce a algunos Estados miembros hacia posiciones más posibilistas en el proceso de negociación y formalización de una propuesta.

Esta afirmación no debe hacernos pensar en el CUE como un foro de ganancias suma-cero por parte de los diferentes actores. Los patrones de interacción dentro del CUE, combinan instruméntos ligados a la diplomacia clásica, con un contexto en el que prima el espíritu cooperativo y donde en pocas ocasiones hay ganadores o perdedores claros. La Presidencia mediante una labor eminentemente política y los propios Estados miembros, se esfuerzan por llegar a acuerdos que satisfagan a todos de alguna manera, utilizando instrumentos como los package deal o las concesiones en alguna otra área donde los Estados en desacuerdo muestren cierto interés ${ }^{47}$.

za el Compromiso de Luxemburgo, pero se muestra pesimista sobre el efecto que puedan tener sobre la eficacia legislativa (tiempo y número), las reformas institucionales que han otorgado más poder al PE y el poder de los Estados miembros para influir en los procedimientos institucionales que rigen el proceso decisorio de la UE, ver GoluB, J.: "In the Shadow of the vote? Decision Making in the European Community", en International Organization, VI, 54, n. ${ }^{\circ} 4,1999$.

46 A este respecto, ver WEILER, J. H. H.: Europa, fin de siglo, Centro de Estudios Constitucionales, Madrid, 1995, pág. 96.

47 Ver WESSELS, W.: "The EC Council:The Community's Decisionmaking Centern, op. cit. Sobre las dinámicas consensuales en el propio CUE, ver NicoLL, 
Ayuda además, el hecho de que el modo de negociación dentro de la UE como complejo político, esté caracterizado por el enfoque hacia la resolución de problemas comunes a través de un contexto negociador de tipo integrativo 48 , lo que indudablemente plantea la necesidad de realizar esfuerzos creativos en la búsqueda de acuerdos que satisfagan a los quince participantes en el CUE y demás actores institucionales (Comisión y PE). Por supuesto, las confrontaciones o negociaciones conflictivas también existen bajo determinadas circunstancias o situaciones ${ }^{49}$.

Algunos autores han señalado que esta forma de tomar decisiones, en la que los miembros del CUE llegan a acuerdos llevando el consenso más allá de lo estrictamente necesario, se traduce en la creación de políticas de una calidad sub-óptima, es decir, no cumplen los estándares mínimos que en realidad requiere la provisión de bienestar vinculada a la legitimidad de los poderes públicos hacia los ciudadanos $^{50}$. Sin embargo, parece evidente que esta situación solamente es probable en los ámbitos o politicas más sensibles al interés nacional. En los ámbitos de cooperación política, como es el caso de la PESC, la existencia del veto y la unanimidad hace difícil no prever la posibilidad de conseguir acuerdos de un rango inferior a lo esperado.

La realidad parece indicar no obstante, que la segmentación sectorial de las políticas, la división del poder decisorio en especialidades funcionales y la naturaleza continuada de los debates políticos, limita la capacidad de las "altas políticas» para condicionar el proceso decisorio de una forma cualitativa y cuantitativa ${ }^{51}$. Además, el sis-

Sir W.: «Representing the States", en DUFF, A.; PINDER, J., Y PRYCE, J.: Maastricht and Beyond: Building the European Union. Routledge, Londres, 1994.

48 Asi lo ve LAFFAN, B.: «The big budgetary bargains: from negotiation to authority", en JEPP, 7: 5, 2000, págs. 741 y 742, y ELGSTRÖM, O., y JöNSSON, C.: "Negotiation in the EU: bargaining or problem-solving?", en JEPP, 7: 5, diciembre de 2000. El contexto negociador al que estos últimos autores hacen referencia, está determinado además por cinco variables: la regla decisora que contenga la base jurídica elegida para tomar la decisión (unanimidad o mayoría), el nivel de politización, el nivel decisorio, el tipo de política y las características de la estructura administrativa u organizativa.

49 ElgstRöm, O., y Jönsson, C., op. cit., págs. 689 y ss.

50 Ver ScharPH, F. W.: «The joint-decision trap: Lessons from German federalism to European integration", en Public Administration, VI, 66, n. ${ }^{\circ} 3,1988$.

51 A este respecto, PETERS, B. G.: «Escaping the Joint-Decision Trap: Repetition and Sectorial Politics in the European Union", en West European Politics, VI, 20, n. ${ }^{\circ} 2,1997$. 
tema decisorio de la UE y el proceso político que lo acompaña, han sido capaces de adaptarse eficazmente a las innovaciones políticas sujetas al cambio de circunstancias externas, que han obligado a tomar efectivas decisiones respecto a cuestiones tan concretas como la inseguridad energética, la polución medioambiental o el crimen transfronterizo ${ }^{52}$.

Por otro lado, la toma de decisiones dentro del CUE, también se ve mediatizada por la despolitización y creciente tecnificación de las materias en las que legisla la propia UE. En ámbitos como el mercado o la moneda única, se suele realizar un planteamiento técnico de problemas eminentemente políticos. Muchos de estos temas son llevados hacia prácticas rutinarias de aprobación de propuestas (temas $A$ en las reuniones del CUE), con las que difícilmente entra en contradicción el interés de los Estados miembros ${ }^{53}$. El COREPER maneja en la mayoria de las ocasiones, propuestas realizadas por grupos de expertos en relación directa con el trabajo de iniciación de la propia Comisión, lo que da cobertura a una conformación tecnocrática de las políticas de la UE y del sistema decisor que las elabora ${ }^{54}$.

Las constantes transformaciones de los diferentes órdenes de la vida social, provocan que la formalización de decisiones se realice bajo altos niveles de incertidumbre, lo que en muchas ocasiones conduce a la formación de grupos de expertos o comunidades epistémicas hacia donde la clase política europea habría desplazado la capacidad de innovación política, definición de prioridades y establecimiento de objetivos ${ }^{55}$. En estas situaciones, tampoco podemos eludir el proble-

52 JORDAN, A.; BROUWER, R., y NOBLE, E.: "Innovative and responsive? A longitudinal analysis of the speed of EU environmental policy-making, 1967-67", en JEPP, 6: 3, septiembre de 1999, pág. 377.

53 Las decisiones "rutinarias» en el ámbito del mercado y la moneda única dentro del CUE, pasaron de un 45\% a más de un 55\% entre los años 1975 y 1986. SLOOT, T., y VERRSCHUREN, P., op. cit., pág. 82, aunque quizá el ejemplo no sea del todo adecuado en la medida que la moneda única y la instauración del Banco Central Europeo, supone en realidad la politización o control político del mercado instaurado tras el AUE.

54 Así lo vieron NoELL, E., y ÉTIENNE, H.: «The Permanent Representatives and the "Deepening" of the Communities", Government and Opposition, 1971, y SPINELLI, A.: The Eurocrats, J. Hopkins University Press, Baltimore, 1966; sobre los grupos de expertos vinculados a la Comisión, ver Peters, B. G.: "Bureaucratic Politics and the Institutions of the European Community", en SBragiA, A. M. (ed.): Bureaucracy and Political Choice, The Brookings Institution, Washington DC, 1992.

55 Sobre este fenómeno, en general, ver HaAS, P.: uIntroduction: Epistemic Communities and International Policy Coordination", en International Organization, 
mático control democrático por parte de la ciudadanía, del proceso político que lleva a la toma de decisiones ${ }^{56}$.

No debemos desdeñar, asimismo, el poder del CUE como una institución generadora de reglas, procedimientos y valores capaces de alterar la relativa influencia de los diferentes actores que participan en el mismo, algo que incluso en ocasiones puede difuminar la distancia entre el interés nacional o comunitario de los propios representantes nacionales que participan en los diferentes niveles del CUE. Participar dentro del proceso decisorio del CUE, puede cambiar la percepción entre lo que es posible y lo que es preferible ${ }^{57}$.

Tanto el COREPER, como los Comités y los grupos de trabajo, se ven envueltos en un proceso decisorio extremadamente complejo, con la difícil misión de representar los intereses de los diferentes Estados y a la vez ser capaces de viabilizar el sistema al que pertenecen a largo plazo. Los participantes dentro de los grupos de trabajo, gracias a la despolitización de muchos temas comunitarios y a la rutinización procedimental, en ocasiones acaban compartiendo intereses comunes como expertos a nivel europeo y generando un espíritu de cuerpo $^{58}$. En este nivel, el desvío de lealtades funcionales e identitarias hacia el ente comunitario, tienen en cualquier caso un valor suplementario 59 , ya que el componente nacional sigue siendo el factor prin-

VI, 46, n. ${ }^{\circ} 1,1992$, y SEBENIUS, J. K.: "Challenging conventional explanations of international cooperation: negotiating analysis and the epistemic communities", en International Organization, VI. 46, n. ${ }^{\circ} 1,1992$. En la UE en particular, de interés VERDUM, A.: wThe role of the Delors Committee in the creation of EMU: an epistemic community", en JEPP, 6: 2, 1999; ZITO, A. R.: "Epistemic communities, collective entrepreneurship and European integration", en JEPP, 8:4, 2001, y BOTCHEVA, L.: "Expertise and International Governance: Eastern Europe and the Adoption of European Union Environmental Legislation", en Global Governance, 7, 2001.

56 Así lo entiende, Cornago Prieto, N.: "Elementos para el análisis del proceso político en los regímenes internacionales: el multilateralismo no necesariamente formalizado", en Anuario de Derecho Internacional, de la Universidad de Navarra, VI, 15, año 1999, pág. 220.

57 Ver KerRemans, B.: “Do Institutions Make a Difference? Non-Institutionalism, Neo-Institutionalism, and the Logic of Common Decision-making in the European Union», en Governance: An International Journal of Policy Administration. VI, 9, n.², 1996, pág. 231

58 GonzAlez SÁNCHEZ, E.: Manual del Negociador en la Comunidad Europea, OID, Madrid, 1992, págs. 81-82.

59 EGEBER, M.: «Transcending Intergovernmentalism? Identity and Role Perceptions of National Officials in EU Decision-Making", Working Paper 98/24 del Instituto sobre la Europeización del Estado, ARENA, http://www.arena.uio.no/, 1998. 
cipal en las relaciones establecidas entre los participantes en los grupos de trabajo 60 .

Los miembros del COREPER han ido conformando con el tiempo, la suerte de un colectivo donde los individuos interactúan a través de implícitas normas de entendimiento, persistentes en el tiempo e independientes de los resultados obtenidos en las negociaciones. La reciprocidad difusa, la confianza y responsabilidad mutua y una arraigada cultura del compromiso, son algunas de las claves para cuestionar el carácter inequivocamente intergubernamental del COREPER ${ }^{61}$. Los Representantes Permanentes son conscientes de la necesidad de mantener el proceso político más allá de los puntos de vista estratégicos o conflictivos, de lo contrario se pondria en peligro la perdurabilidad y viabilidad del sistema al que pertenecen.

Así, gracias a la flexibilidad de unas órdenes provenientes de las administraciones centrales, que se sitúan más en el nivel de las recomendaciones que de las instrucciones, los miembros del COREPER toman decisiones que no siempre se corresponden con el interés nacional que se supone representan ${ }^{62}$ y eluden generalizar la llegada a acuerdos a través de la mayoría cualificada, con el objetivo de contentar finalmente a todos los participantes e intentar no deses-

60 Sin embargo, los actores institucionales tienen una trascendencia destacada en el establecimiento de una red de comunicaciones más o menos intensa entre los participantes en los grupos de trabajo. A este respecto, ver BEYERS, J., y Diericks, G.: «Nationality and European Negotiations:The Working Groups of the Council of Ministers», en European Journal of International Relations, VI, 3, n. ${ }^{\circ}$ 4, 1997; BeYERS, J., y Diericks, G.: "The Working Groups of the Council of the EU: Supranational or Intergovernmental Negotiations?", en $J C M S, V I, 36, n .^{\circ} 3$, 1998.

61 El neofuncionalismo más incipiente, ya había apuntado la dimensión más comunitaria o pro-europea del propio COREPER, así, ver LINBERG, L.: “DecisionMaking and Integration in the European Community", en International Organization, VI, 19, n. ${ }^{\circ} 1,1965$.

62 En este sentido, ya clásicas las reflexiones realizadas por el Representante Permanente alemán, Dietrich von Kyaw, en las que aseguraba que en su capital de origen era coloquialmente denominado como traidor permanente, más que como Representante Permanente de los intereses alemanes. Ver Financial Times, 11/12, marzo de 1995. En la misma línea, de interés las declaraciones de un Representante Permanente sobre la negociación de un asunto en concreto, en LEWIS, J.: "Is the "Hard Bargaining" Image of the Council Misleading? The Committee of Permanent Representatives and the Local Elections Directive", en JCMS, VI. 36, n. ${ }^{\circ} 4$ 1998, pág. 492; También LEWIS, J.: "The institutional Problem Solving Capacities of the Council:The COREPER and the methods of community", Discussion Paper 1/98 del Instituto Max Plank, http://eiop.or.at/erpa/erpaframe.html 
tabilizar un modelo decisorio basado en unos equilibrios tan complejos $^{63}$.

\section{ELTRATADO DE NIZAY EL CUE: EL PROBLEMÁTICO EQUILIBRIO ENTRE EFICACIA Y DEMOCRACIA}

ElTratado de Niza, abordó la necesaria reforma institucional previa a la escalonada pero irremediable incorporación de nuevos Estados al proyecto comunitario a partir del 2005. La descripción y el análisis del contexto previo y la negociación del propio Tratado con respecto al CUE, escapa de las intenciones de este trabajo, por lo tanto, nos centraremos en las sustantivas modificaciones que afectan al proceso decisorio y en la repercusión que pueda tener en el proceso político descrito, en una UE con 27 miembros.

En lo referente al CUE, las dos grandes cuestiones con las que se enfrentaban en la Conferencia Intergubernamental (en adelante CIG) de 2000, se referían a las reglas de votación por este utilizadas para la toma de decisiones y el ámbito material de la regla de la mayoría cualificada. En cuanto a esta última cuestión, el Tratado de Niza ha extendido el ámbito material de la mayoría cualificada a una treintena de casos o artículos; sin embargo, parece que los Estados miembros se resisten a extender la misma en cuestiones tan claves como la fiscalidad, la seguridad social, las cuestiones referidas al asilo e inmigración y los propios fondos estructurales, materias en las que será difícil avanzar en una futura Unión ampliada64. Cabe preguntarse en este caso, si el proceso de integración puede haberse agotado

63 Esta situación tuvo lugar para la aprobación de la Directiva 93/104/EC, referida al tiempo de trabajo, donde la mayoria cualificada fue alcanzada con dos años de antelación, aunque se prefirió adoptarla dos años después por unanimidad, hasta que fue posible alcanzar un acuerdo con el Representante Permanente inglés; los participantes describen este hecho como un "gasto de tiempo extra" pero necesario, "para llevar a todo el mundo dentro del barco». Ver LEWIS, J.: «The methods of community in EU decision-making and administrative rivalry in the Council's infraestructure", op. cit., pág. 271.

64 A este respecto, GutiérRez ESPADA, C.: "Una reforma “difícil pero productiva": La revisión institucional en el Tratado de Nizan, en $R D C E, n .{ }^{\circ} 9$, enero/junio de 2001, pág. 48, y Neunreither, K.: «The European Union in Nice: A Minimalist Approach to a Historic Challenge", en Government and Opposition, VI, 36, n. ${ }^{\circ}$, verano de 2001, pág. 194. 
en lo que respecta a una generalizada extensión material de la mayoría cualificada.

Por otro lado, la CIG de Niza se enfrentó a la difícil misión de compatibilizar la eficacia decisora dentro del CUE, con la adecuación democrática de un sistema de votación que ya a partir de 1995, con la adhesión de Suecia, Austria y Finlandia, ponía en peligro los principios y equilibrios globales que operaban como fuente de legitimidad del CUE entre los participantes ${ }^{65}$. Desde el punto de vista democrático, la cuestión se complicaba más si se pretendía extender el actual sistema de atribución de votos a una UE ampliada a los 27 Estados: entonces una decisión por mayoria cualificada podría alcanzarse por un grupo de Estados que representasen entre, según los casos, el 46,41 y $50,10 \%$ de la población 66 .

ElTratado de Niza ha optado finalmente por un sistema de mayoría cualificada más compleja que la actual. Fijada la nueva ponderación de votos ${ }^{67}$, ésta se alcanza con nuevo sistema de «triple llave»: un número de votos ponderados, que irá creciendo en función del ritmo de adhesiones, y que en una Unión de 27 Estados parece establecerse en 258 votos a favor sobre un total de $345^{68}$, que además representen la mayoría de los Estados miembros que participan en el CUE y que por último comprenda como mínimo el $62 \%$ de la población total de la UE.

Esta reforma, mientras la UE no defina de manera concluyente su naturaleza, otorga más democracia al funcionamiento del CUE res-

65 MANGAS MARTIN, A.: "Democracia y eficacia en la Unión Europea ampliada: el restablecimiento de los equilibrios globales en el sistema de votaciones del Consejon, op. cit., pág. 7.

66 Desde la otra cara, un grupo de Estados podría bloquear una decisión, integrando únicamente el 12 ó $13 \%$ de la población total de la Unión. Comunicación de la Comisión para adaptar las instituciones y reforzar la ampliación con éxito: COM (1999) 592, Bruselas, 2 de diciembre de 1999.

67 Desde el 1 de enero de 2005, la ponderación de votos en el CUE se reforma quedando con los siguientes números: 29 votos para Alemania, Francia, Gran Bretaña e Italia, 27 para España, 13 para Holanda, 12 para Bélgica, Grecia y Portugal, 10 para Austria y Suecia, 7 para Dinamarca, Irlanda y Finlandia y 4 para Luxemburgo. Art. 205.2 y 4 del TCE modificado por el art. 3 del Protocolo para la ampliación, Tratado de Niza (SN 1247/1/01/ Rev, 1, pág. 91), DOCE, 2001/C80/01.

68 Decimos parece, porque ateniéndonos a esta cifra, esto supone un $74^{\prime} 78 \%$ de los votos totales, cuando en realidad la Declaración 21 del Tratado, relativa a la mayoría cualificada y el número de votos de la minoría de bloqueo, establece que uel umbral de la mayoría cualificada evolucionará en función del ritmo de adhesiones, a partir de un porcentaje inferior al actual hasta alcanzar un máximo de $73^{\prime} 4 \%$ ". Tratado de Niza, DOCE, 2001/C80/01, pág. 85. 
pecto de la ciudadania de cada Estado (sobre todo de Alemania, el más perjudicado en la relación actual voto/población dentro del CUE), pero sin embargo modifica de jure y de facto el proceso decisorio dentro del CUE, convirtiéndolo más denso, complejo y probablemente lento ${ }^{69}$. En primer lugar, aunque parezca improbable, se ha señalado que la traumática negociación llevada a cabo en Niza, puede más tarde perpetuar coaliciones dentro del CUE entre diferentes Estados de acuerdo con su tamaño y quizás crear así cleavages o ejes permanentes entre un número de Estados pequeños, medianos o grandes ${ }^{70}$. Asimismo, parece que no se ha caído en la cuenta, de que la creación de un sistema de dobles mayorías (voto y población) para adoptar decisiones dentro del CUE, se va a realizar sobre un aumento en número de Estados que puede llegar casi a doblar el actual. Por lo tanto, teóricamente, se aumenta de manera importante tanto el número de coaliciones posibles ganadoras, como también y sobre todo, el número de coaliciones bloqueadoras en el procedimiento de adopción de decisiones dentro del CUE ${ }^{71}$.

Es cierto, no obstante, que algunos aspectos de la "triple llave» acordada en Niza, ratifican legalmente el hábito negociador dentro del CUE a la hora de tomar decisiones más acá del proceder consensual anteriormente descrito: los Estados pequeños acompañan a los grandes en todas las votaciones en función de sus intereses económicos y sociales, que coinciden siempre, entre otras cosas, según el grado de desarrollo o la apertura de sus economías ${ }^{72}$. Sin embargo, no nos pare-

69 Wessels destaca que tras elTratado de Niza, se distinguen 17 modelos de toma de decisión dentro del CUE, a las que se unen además 11 formas posibles de participación del propio PE (En total, 38 posibles interacciones entre el CUE Y el PE en el marco del proceso decisorio). Ver WESSELS, W.: "Nice Results: The Millennium IGC in the EU's Evolution", en JCMS, VI, 39, n. ${ }^{\circ} 2,2001$, pág. 202. También ver YATAGANAS, $X .:$ "The Teatry of Nice:The sharing of power and the institutional balance in the EU - A continental perspective", en los Working Papers de la Cátedra Jean Monnet de Harvard: W.P. 01/01. http://www.jeanmonnetprogram.org/papers/, año 2001.

70 En este sentido, ver algunas aportaciones realizadas a posteriori por WeSsels, W.: “Nice Results:The Millennium IGC in the EU's Evolution”, op. cit., pág. 206, y con anterioridad por SCHMITTER, P. C.: How to Democratize the European Union... And Why Bother?, CO: Rowman y Littlefield, Boulder, 2000.

71 HosLI, M.: aThe new voting Weights in the Council of the European Union", https://wwwc.cc.columbia.edu/sec/dlc/ciao/isa/isa.html, en los Working Paper de los International Studies Association Network, año 2000, y HosLI, M.: «The balance between small and large: effects of a double-majority system on voting power in the European Union", en International Studies Quarterly, 39, 1995.

72 Elorza, J.: "La UE después de Niza", Política Exterior, n. ${ }^{\circ} 79$, enero/febrero de 2001, pág. 97. 
ce acertado transponer ni procedimental, ni metodológicamente, el proceso político que en la actualidad rodea a la toma de decisiones dentro del CUE, a una Unión con 27 Estados miembros.

No hay que descartar por ejemplo que la red poblacional establecida en el Tratado de Niza, pueda tener un impacto similar al que tuvo en su momento el Compromiso de Luxemburgo, estableciendo un modus operandi en el que los Estados miembros y la Comisión tengan que trabajar bajo premisas de carácter anticipativo, en las que la amenaza de una minoría de bloqueo poblacional, funcione a modo de mecanismo psicológico donde las tomas de posición ante las propuestas estén siempre en función de la satisfacción de una minoría ${ }^{73}$.

Los Estados miembros deberían haber sopesado que estas y otras cuestiones pueden volver al sistema decisorio del Consejo, y por ende de la UE, más lento e ineficaz, disminuyendo entonces su capacidad efectiva como proveedor de bienestar ciudadano a través de la formación de políticas públicas y dañando así uno de los principios o elementos sobre los que se asienta la legitimidad de la propia UE frente a la ciudadania que la conforma ${ }^{74}$.

\section{CONCLUSIONES}

Algunas de las cuestiones abordadas en este artículo, revelan que el CUE es algo más que un foro intergubernamental donde los Estados deciden, de manera simplificada, el modo de incidencia sobre políticas donde han decidido mancomunar o ceder el ejercicio de ciertas competencias ${ }^{75}$. Evidentemente, no aludimos en este caso a una caracterización supranacional del CUE, ya que por un lado, los Estados se han ocupado de establecer mecanismos de "cierre intergubernamental» que no les haga perder el control final sobre el proceso deci-

73 Un criterio bastante parejo al expuesto en torno a esta cuestión, en WESSELS, W.: "Nice Results: The Millennium IGC in the EU's Evolution», op. cit., págs. 206 y 207.

74 Sobre los elementos sobre los que se asienta la legitimidad de la UE, de interés ver SCHARPF, F. W.: “Economic integration, democracy and the welfare state", JEPP, 4: 1, marzo de 1997, págs. 19 y 20, y también HÖRETH, M.: «No way out for the beast? The unsolved legitimacy problem of European governance», en JEPP, 6:2, junio de 1999.

75 Del mismo parecer HAAS, E.: The Uniting of Europe. Political, social and ecomomic forces, 1950-1957, Stanford, Stanford University Press, 1968, y SASSE, C.: Decision-Making in the European Community, Praeger, Nueva York, 1977. 
sorio y de integración, y por otro, el proceso de toma de decisiones en las áreas intergubernamentales, como la PESC, difiere notablemente del aplicable en el ámbito comunitario o definitivamente integrado ${ }^{76}$. Sin embargo, el estudio del CUE y de su proceso decisorio, entendido bien como finalidad superadora o como complemento instrumental del Estado, muestra que en ocasiones los mecanismos intergubernamentales pueden arribar al terreno de lo simbólico y que el proceso de integración comunitaria es capaz de generar dinámicas autónomas.

El proceso decisorio dentro del CUE, se ve mediatizado por diversos factores. Externamente, el PE ha ido adquiriendo un peso legislativo más allá de los difusos controles consultivos o judiciales que ejercitaba sobre el CUE, con anterioridad a la instauración del proceso de codecisión. Por otro lado, el Consejo Europeo conserva poder legislativo efectivo tanto en áreas comunitarizadas, como en ámbitos vinculados a la cooperación intergubernamental. Asimismo, el Consejo Europeo, gracias a su estructura, sigue en condiciones de superar los desequilibrios corporativos vinculados a las diferentes políticas asignadas a los diferentes Consejos y de asumir la dimensión integradora que parece haber disuelto la errónea iniciativa de diferenciar entre "Consejos de cooperación" y "Consejos clásicos»"7.

Internamente, el desarrollo del CUE es parejo y consecuencia de la evolución de Bruselas hacia una mayor complejidad, especialización y fragmentación del proceso de toma de decisiones dentro de la UE. La división funcional-material de tareas en torno al COREPER, grupos de trabajo y los Comités que preparan los diferentes Consejos, pueden hacer perder coherencia y eficacia a la formulación de políticas

76 Debido tanto a la concentración de poderes en el seno del CUE, como al hecho de que los debates y la negociación entre los Estados miembros en dicho marco, no coinciden exactamente con el procedimiento tradicionalmente establecido para la discusión de los temas comunitarios, es decir, debates a nivel de grupos de trabajo, COREPER y el Consejo propiamente dicho; ver GoNZÁLEZ SÁNCHEZ, E.: «El proceso de toma de decisiones en el ámbito de la Política Exterior y de seguridad Común", en $R E D C$, enero-junio de 2001, págs. 411-412.

77 EI PE, en la iniciativa A5-0308/2001, sobre asuntos institucionales, en la que elabora un informe sobre la reforma del Consejo, considera imprescindible que el «Consejo Europeo vuelva a ejercer su función de orientación política, cuestionada por el excesivo número de asuntos de su orden del día y el tiempo dedicado a cuestiones de detalle, lo que se debe a las dificultades crecientes en el funcionamiento del Consejo de Ministros". En Internet, http://www.europarl.es/actualidad/ficha.phtm?p=2\&id=728; Sobre las consecuencias en el sistema institucional, de la diferenciación entre "Consejos de cooperación" y "Consejos clásicos", de interés, MARTINez SierRA, J. M., op. cit., págs. 77-82. 
comunitarias, debido al solapamiento de competencias, a la rivalidad administrativa en pos del logro de recursos y sobre todo a las diferentes percepciones, expresadas en modo de actuación, en torno a los objetivos y significación del sistema en el que participan: la UE.

El análisis exploratorio, muestra que existe una distancia efectiva entre la capacidad formal y material de los Ministros que aprueban las normas a nivel del propio Consejo. En la práctica, el COREPER, los diversos Comités y los grupos de trabajo, asumen un poderoso control sobre el proceso decisorio, gracias a la cantidad de legislación que manejan y de facto aprueban y al conocimiento de un proceso político constituido por una tendencia general a la llegada al consenso, la importante despolitización de los temas tratados y el valor de un contexto institucional que difumina la distancia entre el interés nacional y comunitario, en la búsqueda de funcionalidad a largo plazo del propio sistema comunitario.

El poder legislativo real que asumen los cuerpos burocráticos del CUE, es un obstáculo para mejorar la trasparencia y para que la ciudadanía ejerza, en su caso, un control básico sobre las decisiones que sus representantes ministeriales aprueban dentro del CUE. A las propuestas realizadas con anterioridad ${ }^{78}$, se la puede añadir la necesidad de revalorizar el Consejo de Asuntos Generales y la designación de Ministros con una dedicación exclusiva a asuntos comunitarios, así como la disminución de responsabilidades legislativas por parte del COREPER, a meras funciones de preparación ${ }^{79}$.

Por otro lado, elTratado de Niza parece haber solventado las graves carencias democráticas que asomaban dentro del CUE, en el caso de seguir aplicando el sistema actual de reponderación de voto, a una UE con más de 25 Estados. Sin embargo, a nuestro entender, son necesarias la adopción de una serie de medidas que permitan al CUE seguir siendo una institución eficaz como generadora de acuerdos en forma de legislación. Los Estados miembros deben de plantearse la necesidad de crear una Presidencia permanente que asegure continuidad, coherencia y perspectiva integradora a la acción comunitaria, la articulación flexible de las sesiones plenarias de un Consejo con casi el doble de participantes y la clara distinción entre las labores legislativas y ejecutivas en la relación del CUE con otras instituciones.

78 Vid. infra, cita 42.

79 Del mismo parecer el PE, en la Iniciativa A5-0308/2001, sobre asuntos institucionales. En Internet http://www.europarl.es/actualidad/ficha.phtm? $p=2 \&$ id $=728$ 
La perspectiva más pesimista indica que la adhesión de nuevos Estados puede intergubernamentalizar el proceso político antes descrito, si bien algunos pensamos que por el contrario, tal cuestión, puede tener un efecto federalizante sobre el proceso decisorio dentro del CUE. Los nuevos Estados miembros pueden verse imbuidos dentro de un gran sistema político y económico, en la que ente la perspectiva de verse marginados o fuera de la propia dinámica organizativa, opten por integrarse a través de los patrones de interacción establecidos $Y$ asentados dentro del CUE durante años. 